\title{
2. RED SUBLUMINOUS STARS
}

\section{O. J. EGGEN}

Mount Stromlo and Siding Spring Observatories, Research School of Physical Sciences, The Australian National University, Camberra, Australia

(UBVRI) observations of known subluminous stars were published in a previous discussion (Eggen, 1970a) where the bulk of the objects, both red (RSL) and blue (BSL) populated a sequence extending for $\mathrm{M}(\mathrm{I})$ near $+11^{\mathrm{m}} 5$ at $(\mathrm{R}-\mathrm{I})$ near 0.0 to $\mathrm{M}(\mathrm{I})$ of $+15^{\mathrm{m}}$ near $\mathrm{R}-\mathrm{I}=+0^{\mathrm{m}} 3$. However, four RSL stars appeared to form a separate sequence near $M(I)=+11^{\mathrm{m}}$ and $\mathrm{R}-\mathrm{I}$ between $+0 \mathrm{~m} 3$ and $+0 \mathrm{~m}$. These four stars, plus an additional object (LTT 2236) are listed in Table I together with the source of luminosity; the weights of the trigonometric parallaxes are given in parentheses following the parallax. These five stars are represented in Figure 1 by crosses; the top of the steep sequence of subluminous stars is shown as the hatched region in the figure. For reference, Figure 1 also contains (1) the Hyades main sequence (Eggen, 1969b), indicated by a broken curve, (2) the evolved main sequence of the old disk population (Eggen, 1970b), shown by open circles, and (3) the subdwarfs, which are listed in

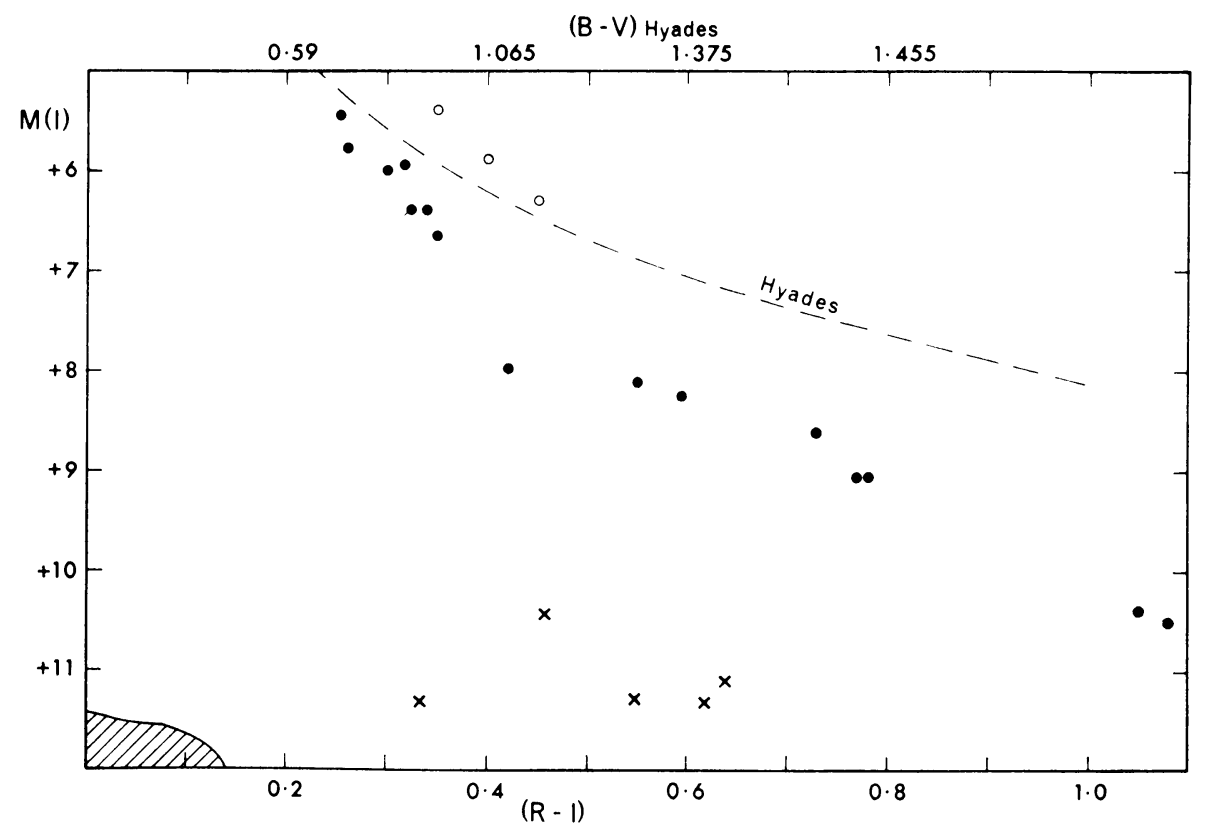

Fig. 1. Evolved, old disk population main sequence stars (open circles), Hyades cluster stars (broken curve), subdwarfs (filled circles, and RSL stars (crosses) in the $\left(\mathrm{M}_{I}, \mathrm{R}-\mathrm{I}\right)$ plane. The top of the steep sequence of subluminous stars (white dwarfs) is shown as the hatched area in the lower left hand corner. 


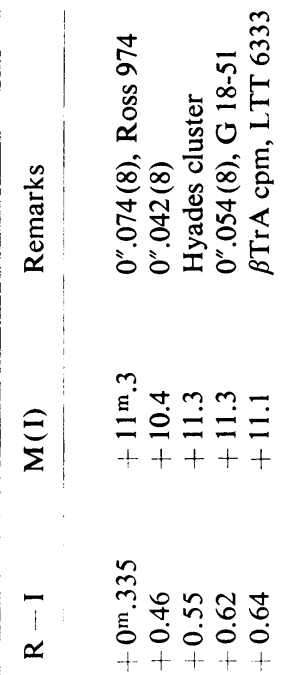

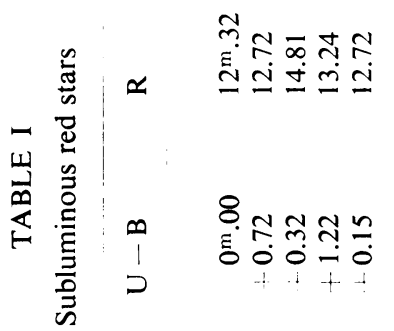

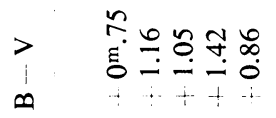

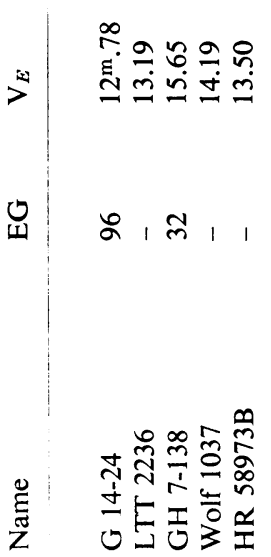


TABLE II

Late type subdwarfs

\begin{tabular}{lrrrrrrrr}
\hline Yale & \multicolumn{1}{c}{$\mathrm{V}_{E}$} & $\mathrm{~B}-\mathrm{V}$ & $\mathrm{U}-\mathrm{B}$ & $\mathrm{R}$ & $\mathrm{R}-\mathrm{I}$ & $\mathrm{M}(\mathrm{I})$ & $\pi_{t r}(\mathrm{wt})$ \\
\cline { 7 - 9 } & & & & & & & \\
887.0 & 8.51 & +0.86 & +0.37 & 8.10 & +0.34 & +6.4 & $0.053(58)$ \\
948.1 & 11.85 & +1.37 & +1.02 & 10.90 & +0.37 & +8.6 & $0.048(12)$ \\
1181.0 & 8.90 & +1.53 & +1.07 & 7.84 & +0.77 & +9.05 & $0.251(18)$ \\
1857.0 & 8.32 & +0.62 & -0.14 & 8.15 & +0.26 & +5.8 & $0.038(61)$ \\
2392.0 & 8.07 & +0.595 & -0.025 & 7.68 & +0.255 & +5.45 & $0.040(16)$ \\
2512.0 & 11.02 & +1.40 & +0.95 & 10.04 & +0.775 & +9.05 & $0.091(7)$ \\
2745.0 & 6.45 & +0.75 & +0.17 & 6.08 & +0.30 & +6.0 & $0.110(36)$ \\
3044.0 & 10.85 & +1.00 & +0.68 & 10.23 & +0.42 & +8.0 & $0.043(15)$ \\
3252.0 & 13.43 & +1.58 & +1.08 & 12.21 & +1.05 & +10.4 & $0.070(12)$ \\
$3425.0 \mathrm{~A}$ & 9.10 & +0.78 & +0.13 & 8.71 & +0.32 & +6.4 & $0.040(35)$ \\
$3425.0 \mathrm{~B}$ & 9.45 & +0.85 & +0.30 & 9.00 & +0.35 & +6.65 & \\
3669.0 & 7.52 & +0.84 & +0.24 & 7.20 & +0.32 & +5.9 & $0.063(40)$ \\
3783.1 & 12.73 & +1.64 & +1.20 & 11.63 & +1.08 & +10.5 & $0.099(10)$ \\
$5741.1 \mathrm{~A}^{*}$ & 12.13 & +1.28 & +1.05 & 11.26 & +0.55 & +8.1 & $0.030(*)$ \\
$5741.1 \mathrm{~B}{ }^{*}$ & 12.84 & +1.39 & +1.16 & 11.39 & +0.595 & +8.2 &
\end{tabular}

* The values for the bright and faint components are, respectively, $0^{\prime \prime} .024(14)$ and $0^{\prime \prime} .042(7)$.

Table II. In addition to the photometric results, Table II contains the Yale Parallax Catalogue number, the mean trigonometric parallax and its weight, in parentheses, as well as the resulting values of $\mathrm{M}(\mathrm{I})$.

The luminosity of any one star in Table I cannot be accepted without some reserve.

(1) The single trigonometric parallax, determined at the Cape, for G 14-24 is large and apparently (Greenstein and Eggen, 1966) well determined. The total annual proper motion is 0.47 , leading to a tangential velocity of $275 \mathrm{~km} / \mathrm{sec}$ if the star were a subdwarf $(\pi=0.008)$. The radial velocity is $+156 \mathrm{~km} / \mathrm{sec}$ so the resulting total space motion of $320 \mathrm{~km} / \mathrm{sec}$ does not eliminate the possibility that this is a subdwarf.

However, the requirement that the trigonometric parallax is in error by a factor of 10 is very unlikely. The spectrum of the star is indistinguishable from that of a weak-lined subdwarf (Eggen and Greenstein, 1965).

(2) The single trigonometric parallax of 0.042 (wt. 8) for LTT 2236 has been determined at the Cape Observatory (Yale, No. 1216.1). If the star is a subdwarf the parallax is 0.012 and the total annual proper motion of 0.52 would give a tangential velocity of $200 \mathrm{~km} / \mathrm{sec}$. Additional parallax determinations are needed.

(3) The membership of GH 7-138 (van Altena, No. 71) in the Hyades cluster seems well established on the basis of three determinations of the proper motion (van Altena, 1969; Table IIIc).

(4) The single trigonometric parallax determination for Wolf 1037 (LTT 16591) is from the Mount Wilson Observatory. As a subdwarf the parallax would be 0."015 and the large annual proper motion of 1."68 would lead to a tangential velocity of $530 \mathrm{~km} / \mathrm{sec}$. The radial velocity of $-160 \mathrm{~km} / \mathrm{sec}$, which may be variable, gives a total space motion of $550 \mathrm{~km} / \mathrm{sec}$. As for G 14-24, the spectrum is indistinguishable from 
that of a very weak-lined subdwarf, K-type star (Joy, 1947). Additional parallax observations are needed.

(5) Spectra of LTT 6333 are not available. The luminosity is based on the assumption that the proper motion of LTT $6333\left(0^{\prime \prime} 40223^{\circ}\right)$ is common with that of HR 5897 $\left(\beta \operatorname{Tr} \mathrm{A}, 0.42205^{\circ}\right)$ which is $157^{\prime \prime}$ distant. The single trigonometric parallax determination for HR 5897 is 0.078 (wt. 7) from the Cape Observatory and the photometric value, from $(u, b, v, y)$ photometry is 0.063 .

The five late type, subluminous stars discussed above also show abnormal values of the ultraviolet excess. This is demonstrated in Figure 2(a) where the continuous

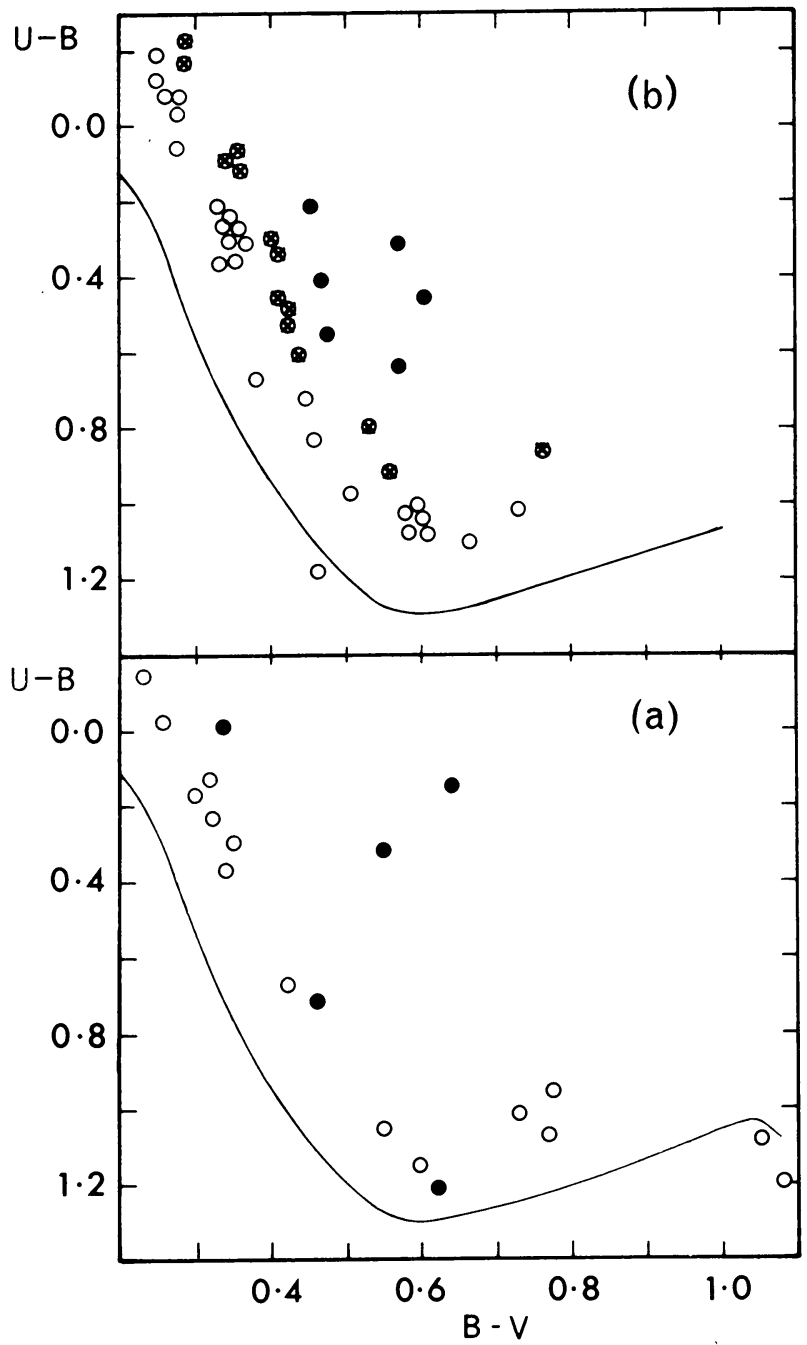

Fig. 2. The continuous curve in both panels represents the main sequence stars of the Hyades cluster. The open and filled circles in (a) represent the subdwarfs and subluminous stars in Tables II and I, respectively. The open circles in (b) represent probable subdwarfs (sd?) in Table III, the crossed circles, possible RSL stars and the filled circles, probable RSL stars. 
TABLE III

Possible RSL stars

Star

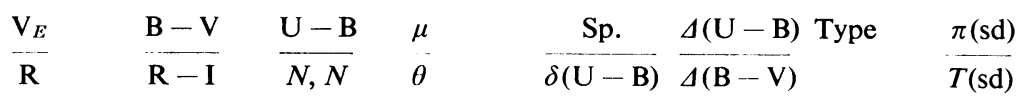

\begin{tabular}{|c|c|c|c|c|c|c|c|c|}
\hline L 233-10 & 14.46 & +0.835 & +0.22 & $0^{\prime \prime} .21$ & g & +0.235 & RSL & $0^{\prime \prime} .007$ \\
\hline LTT 929 & 14.00 & +0.465 & 3,2 & $173^{\circ}$ & +0.29 & +0.90 & & 150 \\
\hline L 729-4 & 13.26 & +0.985 & +0.40 & 0.46 & k & +0.095 & RSL? & 0.010 \\
\hline LTT 1244 & 12.82 & +0.41 & 2,2 & 170 & +0.40 & +0.575 & & 200 \\
\hline L $127-50$ & 15.20 & +0.95 & +0.27 & 0.24 & k & +0.04 & sd? & 0.003 \\
\hline LTT 1346 & 14.80 & +0.36 & 2,3 & 178 & +0.47 & +0.545 & & 400 \\
\hline L $298-26$ & 13.73 & +0.91 & +0.30 & 0.38 & $\mathrm{~g}$ & +0.09 & sd? & 0.006 \\
\hline LTT 1358 & 13.30 & +0.365 & 2,2 & 90 & 0.36 & +0.53 & & 300 \\
\hline LTT 1561 * & 14.49 & +0.92 & +0.25 & 0.31 & $\mathrm{k}$ & +0.05 & sd? & 0.004 \\
\hline G77-43 & 14.11 & +0.345 & 3,3 & $149^{\circ}$ & +0.43 & +0.505 & & 350 \\
\hline L 54-9 & 13.64 & +0.47 & -0.22 & 0.24 & g & +0.34 & RSL? & 0.0035 \\
\hline LTT 1607 & 13.40 & +0.28 & 3,1 & 84 & +0.23 & +0.635 & & 300 \\
\hline LTT $1721^{*}$ & 14.12 & +0.825 & +0.07 & 0.27 & $\mathrm{k}$ & +0.155 & RSL? & 0.0045 \\
\hline G 160-6 & 13.78 & +0.355 & 2,2 & 190 & +0.415 & +0.73 & & 300 \\
\hline Ross $580^{*}$ & 13.04 & +0.79 & +0.08 & 0.23 & $\mathrm{k}-\mathrm{m}$ & +0.18 & RSL? & 0.0075 \\
\hline G $160-8$ & 12.58 & +0.35 & 2,2 & 150 & +0.33 & +0.70 & & 150 \\
\hline L 807-30 & 14.23 & +1.26 & +0.92 & 0.44 & $\mathrm{~g}$ & +0.09 & RSL? & 0.011 \\
\hline LTT 2030 & 13.53 & +0.56 & 2,3 & 155 & - & +0.36 & & 200 \\
\hline L $665-68$ & 12.95 & +0.67 & -0.08 & 0.40 & $\mathrm{k}$ & +0.105 & sd? & 0.004 \\
\hline LTT 2175 & 12.75 & +0.265 & 3,3 & 138 & +0.285 & +0.43 & & 450 \\
\hline Strand $11^{*}$ & 13.28 & +1.015 & +0.48 & $0^{\prime \prime} .32$ & - & +0.08 & RSL? & $0^{\prime \prime} .010$ \\
\hline G 99-8 & 12.78 & +0.42 & 2,2 & 72 & +0.39 & +0.52 & & 150 \\
\hline Strand 12 & 14.83 & +1.20 & +1.19 & 0.34 & - & -0.01 & ? & \\
\hline G 99-9 & 13.80 & +0.475 & 1,2 & 77 & - & -0.05 & & \\
\hline G $99-47$ & 14.08 & +0.61 & -0.11 & 1.07 & - & +0.165 & sd? & 0.0025 \\
\hline- & 13.83 & +0.25 & 4,3 & 208 & 0.25 & +0.39 & & 2000 \\
\hline L 595-22 & 12.25 & +0.38 & -0.25 & 0.32 & - & +0.09 & * & $*$ \\
\hline LTT 2415 & 12.20 & +0.155 & 5,2 & 124 & +0.27 & +0.265 & & \\
\hline L $812-11$ & 13.14 & +1.00 & +0.53 & 0.88 & $\mathrm{~g}$ & +0.095 & RSL? & 0.011 \\
\hline LTT 2535 & 12.58 & +0.42 & 4,4 & 140 & +0.31 & +0.47 & & 350 \\
\hline L $455-129$ & 13.66 & +1.34 & +1.04 & 0.86 & $f:$ & +0.035 & sd? & 0.012 \\
\hline LTT 2826 & 13.62 & +0.605 & 2,2 & 154 & - & +0.25 & & 450 \\
\hline G $112-28$ & 13.72 & +1.12 & +0.82 & 1.01 & - & +0.05 & sd? & 0.011 \\
\hline - & 13.10 & +0.465 & 3,2 & 169 & - & +0.295 & & 450 \\
\hline G 113-36 & 15.45 & +1.14 & +0.64 & 0.30 & - & +0.215 & RSL & 0.0065 \\
\hline- & 14.73 & +0.57 & 1,3 & 141 & - & +0.645 & & 200 \\
\hline LTT $3144^{*}$ & 11.97 & +0.93 & +0.51 & 1.00 & $\mathrm{k}-\mathrm{m}$ & +0.155 & RSL? & $0.018^{*}$ \\
\hline G 113-40 & 11.41 & +0.41 & 3,3 & 157 & +0.19 & +0.465 & & 250 \\
\hline L 190-19 & 13.30 & +1.00 & +0.675 & 0.39 & $\mathrm{~g}$ & +0.025 & sd? & 0.0085 \\
\hline LTT 3807 & 12.73 & +0.38 & 2,3 & 272 & +0.165 & +0.205 & & 200 \\
\hline L $36-61$ & 13.08 & +1.12 & +0.79 & 0.43 & $\mathrm{k}$ & +0.18 & RSL? & 0.0185 \\
\hline LTT 3862 & 12.22 & +0.53 & 1,1 & 121 & - & +0.46 & & 100 \\
\hline L 260-53 & 13.27 & +1.47 & +1.10 & $1^{\prime \prime} .11$ & $\mathrm{~g}$ & -0.08 & sd? & $0^{\prime \prime} .024$ \\
\hline LTT 5622 & 12.55 & +0.67 & 1,2 & 249 & - & +0.19 & & 200 \\
\hline L 477-3 & 11.90 & +0.88 & +0.28 & 0.52 & $\mathrm{~g}:$ & +0.06 & sd? & 0.011 \\
\hline LTT 5864 & 11.62 & +0.335 & 2,2 & 245 & +0.32 & +0.43 & & 200 \\
\hline L $478-87$ & 13.24 & +0.925 & +0.365 & 0.26 & a-f & +0.055 & sd? & 0.007 \\
\hline LTT 5889 & 12.88 & +0.355 & 2,2 & 270 & 0.335 & -0.43 & & 200 \\
\hline
\end{tabular}


Table III (continued)

\begin{tabular}{|c|c|c|c|c|c|c|c|c|}
\hline \multirow[t]{2}{*}{ Star } & $\mathrm{V}_{E}$ & $B-V$ & $U-B$ & $\mu$ & Sp. & $\Delta(\mathrm{U}-\mathrm{B})$ & Type & $\pi(\mathrm{sd})$ \\
\hline & $\mathbf{R}$ & $\mathbf{R}-\mathbf{I}$ & $N, N$ & $\theta$ & $\delta(\mathrm{U}-\mathrm{B})$ & \multicolumn{2}{|l|}{$\Delta(\mathrm{B}-\mathrm{V})$} & $T(\mathrm{sd})$ \\
\hline L $262-45$ & 13.80 & +0.92 & +0.31 & 0.32 & f & +0.145 & RSL? & 0.007 \\
\hline LTT 5944 & 13.34 & +0.40 & 2,1 & 213 & $\stackrel{-L .37}{0}$ & +0.64 & & 200 \\
\hline Ross $1038^{*}$ & 12.33 & +0.765 & +0.055 & 0.96 & $\mathrm{~m}$ & +0.025 & sd? & 0.0065 \\
\hline G $15-13$ & 12.05 & +0.275 & 2,1 & 207 & +0.31 & +0.355 & & 700 \\
\hline L 624-39 & 12.20 & +0.845 & +0.105 & 0.39 & $\mathrm{~g}$ & +0.115 & sd? & 0.010 \\
\hline LTT 6307 & 11.91 & +0.345 & 2,3 & 186 & +0.425 & +0.65 & & 200 \\
\hline L $628-48$ & 12.90 & +0.68 & -0.16 & 0.30 & f & +0.11 & RSL? & 0.0045 \\
\hline LTT 6447 & 12.78 & +0.275 & 3,2 & 231 & +0.385 & +0.57 & & 300 \\
\hline$-3^{\circ} 3968 A^{*}$ & 9.63 & +0.74 & +0.09 & 0.75 & G5 & +0.17 & RSL? & $0.031^{*}$ \\
\hline G $17-25$ & 9.24 & +0.32 & 4,6 & 193 & +0.23 & +0.555 & & 100 \\
\hline$-33968 \mathrm{~B}$ & 13.88 & +1.43 & 1.46: & 0.73 & - & -0.05 & $?$ & \\
\hline G $17-27$ & 13.01 & +0.63 & 2,4 & 190 & - & -0.18 & & \\
\hline G $17-28$ & 14.28 & +1.42 & +1.07 & 1.26 & - & -0.045 & sd? & 0.012 \\
\hline- & 13.67 & +0.605 & 2,3 & 226 & - & +0.22 & & 500 \\
\hline G $19-17$ & 14.67 & +1.42 & +0.46 & 0.28 & - & -0.045 & RSL & 0.011 \\
\hline- & 13.86 & +0.605 & 1,3 & 189 & - & +0.83 & & 100 \\
\hline L $754-45^{*}$ & 11.52 & +0.535 & -0.19 & $0^{\prime \prime} .24$ & g & +0.195 & sd? & $0^{\prime \prime} .0085$ \\
\hline LTT 3966 & 11.13 & +0.255 & 3,2 & 253 & +0.255 & +0.495 & & 150 \\
\hline L 754-46 & 13.10 & +0.84 & +0.23 & 0.24 & $\mathrm{~g}$ & +0.13 & sd? & 0.0085 \\
\hline LTT 3967 & 12.52 & +0.35 & 5,2 & 253 & 0.29 & +0.55 & & 150 \\
\hline G 163-59 & 14.86 & +1.46 & +0.86 & 1.14 & - & -0.035 & RSL? & 0.016 \\
\hline- & 13.84 & +0.77 & 1,2 & 202 & - & +0.44 & & 350 \\
\hline L $611-42$ & 12.78 & +0.56 & -0.18 & 0.31 & $\mathrm{~g}:$ & - & & \\
\hline LTT 4210 & - & - & $4,-$ & 287 & +0.27 & - & & \\
\hline L $611-43$ & 14.94 & +1.00 & +0.54 & 0.31 & $\mathrm{~g}:$ & +0.355 & RSL & 0.009 \\
\hline LTT 4211* & 14.05 & +0.57 & 5,1 & 287 & +0.30 & +0.745 & & 150 \\
\hline L 614-137 & 14.30 & +1.36 & +1.02 & 0.60 & $\mathrm{k}$ & +0.005 & sd? & 0.0115 \\
\hline LTT 4667 & 13.52 & +0.585 & 2,2 & 154 & - & 0.265 & & 250 \\
\hline L 105-2 & 13.10 & +0.695 & -0.04 & 0.24 & $\mathrm{a}$ & +0.115 & sd? & 0.005 \\
\hline LTT 4896 & 12.80 & +0.28 & 2,2 & 300 & +0.295 & +0.475 & & 250 \\
\hline L $328-123$ & 14.25 & +1.08 & +0.62 & 0.45 & $\mathrm{k}$ & +0.04 & RSL? & 0.0065 \\
\hline LTT 4953 & 13.84 & +0.435 & 3,2 & 296 & 0.35 & +0.42 & & 350 \\
\hline LTT 5074* & 12.94 & +0.95 & +0.30 & 0.58 & $\mathrm{~g}$ & +0.02 & sd? & 0.0085 \\
\hline G 14-39 & 12.40 & +0.35 & 3,2 & 288 & +0.44 & +0.48 & & 350 \\
\hline L 196-36 & 12.28 & +1.36 & +1.08 & 0.30 & g & +0.01 & $\mathrm{sd} ?$ & 0.031 \\
\hline LTT 5220 & 11.49 & +0.59 & 2,2 & 165 & - & +0.31 & & 50 \\
\hline L 547-141 & 12.69 & +0.87 & +0.23 & 0.47 & $\mathrm{k}$ & +0.07 & sd? & 0.008 \\
\hline LTT 5472 & 12.25 & +0.335 & 3,3 & 270 & +0.35 & +0.48 & & 250 \\
\hline L 836-104 & 13.65 & +0.73 & -0.07 & 0.25 & $\mathrm{k}$ & +0.03 & sd? & 0.0035 \\
\hline LTT 5560 & 13.42 & +0.265 & 2,2 & 201 & +0.375 & +0.43 & & 350 \\
\hline Ross 858 & 13.64 & +1.31 & +1.04 & $0^{\prime \prime} .61$ & $\mathrm{k}$ & +0.06 & $\mathrm{sd} ?$ & $0^{\prime \prime} .018$ \\
\hline LTT 6979 & 12.82 & +0.595 & 2,3 & 210 & - & +0.25 & & 150 \\
\hline LTT $7424 *$ & 12.86 & +1.00 & +0.56 & 0.31 & g & +0.19 & RSL & 0.0145 \\
\hline G $155-35$ & 12.42 & +0.475 & 2,2 & 214 & +0.28 & +0.58 & & 100 \\
\hline L 1143-61* & 13.76 & +0.87 & +0.37 & 0.59 & $\mathrm{k}$ & +0.07 & sd? & 0.005 \\
\hline G $25-1$ & 13.37 & +0.335 & 2,2 & 197 & +0.21 & +0.34 & & 600 \\
\hline Ross $770^{*}$ & 11.75 & +1.19 & +0.97 & 1.10 & K4 & +0.07 & sd? & 0.031 \\
\hline LTT 8417 & 11.00 & 0.51 & 5,4 & 192 & - & $\therefore 0.245$ & & 150 \\
\hline L 716-108 & 13.76 & $\div 1.32$ & $\therefore 1.00$ & 1.05 & $\mathrm{~m}$ & +0.05 & sd? & 0.016 \\
\hline LTT 8975 & 12.87 & $\div 0.59$ & 1,3 & 156 & -- & 0.29 & & 250 \\
\hline
\end{tabular}


Table III (continued)

\begin{tabular}{lcccccccc}
\hline Star & $\mathrm{V}_{E}$ & $\mathrm{~B}-\mathrm{V}$ & $\mathrm{U}-\mathrm{B}$ & $\mu$ & $\mathrm{Sp}$. & $\Delta(\mathrm{U}-\mathrm{B})$ & Type & $\pi(\mathrm{sd})$ \\
& $\mathrm{R}$ & $\mathrm{R}-\mathrm{I}$ & $N, N$ & $\theta$ & $\delta(\mathrm{U}-\mathrm{B})$ & $\Delta(\mathrm{B}-\mathrm{V})$ & & $T(\mathrm{sd})$ \\
& & & & & & & & \\
& & & & & & & & \\
LTT 9372* & 13.57 & +1.00 & +0.42 & 0.40 & $\mathrm{k}$ & +0.17 & $\mathrm{RSL}$ & 0.0155 \\
G 157-20 & 13.16 & +0.465 & 3,2 & 115 & +0.42 & +0.695 & & 150 \\
L 793-57 & 13.52 & +0.88 & +0.34 & 0.75 & $\mathrm{~g}$ & +0.07 & $\mathrm{sd} ?$ & 0.0055 \\
LTT 9765 & 13.05 & +0.34 & 7,4 & 169 & +0.26 & +0.39 & & 650 \\
\hline
\end{tabular}

*LTT 1561: $\quad$ Lowell, $\mu=0^{\prime \prime} .31, \theta=139^{\circ}$.

LTT 1721: Lowell, $\mu=0^{\prime \prime} .31,158^{\circ}$.

Ross 580: $\quad$ LTT 1728. Lowell, $\mu-0^{\prime \prime} .28, \theta-149^{\circ}$.

Strand 11/12: Lowell, $\mu=0^{\prime \prime} .29$ and $0^{\prime \prime} .29, \theta=78^{\circ}$ and $78^{\circ}$.

LTT 2415: Almost certainly a subluminous star.

LTT 3144: Lowell, $\mu=0^{\prime \prime} .96, \theta=156^{\circ}$. Trigonometric parallax (Y2019.0) is $0^{\prime \prime} .045, w t=15$.

LTT 3966/7: LDS $315,27^{\prime \prime} 300^{\circ}$.

LTT 4210/1: LDS $350,40^{\prime \prime} 256^{\circ}$.

LTT 5074: $\quad$ Lowell, $\mu=0^{\prime \prime} .59, \theta=290^{\circ}$.

Ross 1038: Lowell, $\mu=0^{\prime \prime} .87, \theta=213^{\circ}$.

$-3^{\circ} 3968 \mathrm{~A}$ : Also LTT $6621, \mu=0^{\prime \prime} .82, \theta=191^{\circ}$. The two stars are separated by $20^{\prime}$. The trigonometric parallax (Y3767.0) is $0^{\prime \prime} .046, w t=17$.

LTT 7424: $\quad$ Lowell, $\mu=0^{\prime \prime} .30, \theta=225^{\circ}$.

L 1143-61: Lowell, $\mu=0^{\prime \prime} .66, \theta=198^{\circ}$. Also G 141-35, $\mu=0^{\prime \prime} .61, \theta=199^{\circ}$.

Ross 770: The trigonometric parallax (Y5100.0) is $0^{\prime \prime} .008, w t=6$.

LTT 9372: Lowell, $\mu=0^{\prime \prime} .49, \theta=116^{\circ}$.

curve represents the Hyades cluster stars and the open circles represent the subdwarfs in Table II. The RSL stars in Table $I$ are shown in Figure $2 \mathrm{a}$ as filled circles and the resulting values of $\Delta(\mathrm{U}-\mathrm{B})=+0 .{ }^{\mathrm{m}} 8,+1 \mathrm{.}^{\mathrm{m}} 0$ and $+1^{\mathrm{m}}{ }^{1} 1$ for $\mathrm{G} \mathrm{12-24,} \mathrm{GH} \mathrm{7-138}$ and HR 5897B, respectively, are considerably larger than expected from the abundance effect alone.

The high ultraviolet (HUV) excess criterion for isolating possible RSL stars was previously applied to some 1000 southern proper motion stars (Eggen, 1969a), About 100 objects, or 10 percent of the proper motion stars, were found to be probably subluminous by this criterion. Observations of $R$ and $R-I$ are now available for 51 of these stars and are listed in Table III together with (1) the discoverer's number and the identifications in the LTT catalogue (Luyten, 1957) or lists published by Giclas and his associates (Lowell Observatory Bulletins); (2) the (UBV) photometry and the number (N, N) of (UBV) and (R, I) observations, respectively; (3) the annual proper motion and its direction; (4) the spectral type or color class (Luyten, 1957) and the ultraviolet excess, $\delta(\mathrm{U}-\mathrm{B}) ;(5)$ the values of $\Delta(\mathrm{U}-\mathrm{B})$ and $\Delta(\mathrm{B}-\mathrm{V})$ obtained from the $(\mathrm{R}-\mathrm{I}, \mathrm{B}-\mathrm{V})$ and $(\mathrm{R}-\mathrm{I}, \mathrm{U}-\mathrm{B})$ relations for Hyades cluster stars (Eggen, 1970b); (6) the probable classification of the star, discussed below, and (7) the photometric parallax and resulting tangential velocity if the star is assumed to a subdwarf and falling on the mean subdwarf sequence in Figure 1. 
The stars in Table III are shown in the $(\mathrm{R}-\mathrm{I}, \mathrm{U}-\mathrm{B})$ plane of Figure $2 \mathrm{~b}$ where the continuous curve represents the relation for Hyades cluster, main sequence stars. The open circles in Figure $2 b$ represent objects that, by analogy with Figure $2 a$, could be subdwarfs, the crossed circles are possible subluminous stars on the basis of the large ultraviolet excesses and the filled circles represent HUV objects that are probably subluminous. The filled circles are referred to as RSL objects in the penultimate column of Table III, the crossed circles as RSL? stars and the open circles as sd? stars. From a comparison with Figure $2 \mathrm{a}$ it is assumed that the sd? stars may include a few RSL objects, the crossed circles may include a few subdwarfs and the filled circles may all represent RSL stars.

The distribution of tangential velocities listed in the last column of Table III and based on the assumption that all of the stars are subdwarfs, adds some weight to the classification of six objects (filled circles in Figure 2b) as RSL stars. Omitting one obvious subluminous star, G 99-47, for which the subdwarf assumption leads to the improbable tangential velocity of $2000 \mathrm{~km} / \mathrm{sec}$, the mean value for the 26 'sd?' stars, or common proper motions systems, is $T=300 \mathrm{~km} / \mathrm{sec}$ compared with $140 \mathrm{~km} / \mathrm{sec}$ as the mean for the 6RSL stars. That is, the stars assigned to the RSL classification on the basis of the ultraviolet excess almost certainly have smaller tangential velocities than the probable subdwarfs. The known RSL stars in Table I have a mean tangential velocity of $40 \mathrm{~km} / \mathrm{sec}$ and the six stars shown as filled circles in Figure $2 \mathrm{~b}$ also give a mean of $40 \mathrm{~km} / \mathrm{sec}$ if the same mean luminosity, $M(\mathrm{I})=+11 \mathrm{~m} 1$, is assumed. The 'RSL?' stars, crossed circles in Figure 1b, give an intermediate value of $230 \mathrm{~km} / \mathrm{sec}$ as the mean tangential velocity on the subdwarf assumption. Some of these stars are undoubtedly RSL stars and from a consideration of both the tangential velocity and the color, the most likely candidates in this group are Ross 580, G 99-8/9, LTT 3862 and $-3^{\circ} 3968 \mathrm{~A}, \mathrm{~B}$. The trigonometric parallax listed in the notes to Table III for this last pair places the fainter component among the RSL stars, with $M(I)=+10$. 7 at $R-I=+0 .{ }^{m} 63$, but gives the brighter component values of $M(I)=+7.2$ at $R-I=$ +0 . 32 . Spectroscopic observations would be of great interest.

TABLE IV

Photometric parallaxes for probable RSL stars

\begin{tabular}{|c|c|c|c|c|c|}
\hline \multirow[t]{2}{*}{ Name } & $\mathbf{M}(\mathrm{I})=$ & & Name & $M(I)=$ & \\
\hline & +11.1 & +14.5 & & +11.1 & +14.5 \\
\hline LTT 929 & $0^{\prime \prime} .031$ & - & LTT 5220 & $0^{\prime \prime} .110$ & - \\
\hline Ross 580 & 0.060 & 0.280 & Ross 1038 & 0.072 & 0.350 \\
\hline G 99-8 & 0.055 & - & $-3^{\circ} 3968$ & See text & - \\
\hline G 99-47 & 0.031 & 0.150 & LTГ 7424 & 0.070 & - \\
\hline LTT 2415 & 0.060 & $0.150 *$ & LTT 9372 & 0.048 & - \\
\hline LTT 3862 & 0.075 & - & LTT 9765 & 0.048 & 0.230 \\
\hline LTT 4211 & 0.033 & - & & & \\
\hline
\end{tabular}


The most likely RSL stars in Table II are listed in Table IV together with the photometric parallax based on the assumption that, like the stars in Table I, the luminosities are near $\mathrm{M}(\mathrm{I})=+11^{\mathrm{m}} 1$. The stars in Table IV also include the three 'sd?' objects in Table III with tangential velocities greater than $600 \mathrm{~km} / \mathrm{sec}$ from the subdwarf assumption (G 99-47, Ross 1038 and LTT 9765). As RSL stars the five objects in Table IV with $(\mathrm{R}-\mathrm{I})$ less than $+0 \mathrm{~m} 35$ may populate the steeper sequence of subluminous stars (white dwarfs) for which the luminosities would be near $M(I)=+14.5$ and in these cases the resulting parallax is also listed in the table.

All of the stars in Tables I and III are of interest but astrometric and spectroscopic observations of those in Tables I and IV are especially important and will probably settle the questions of the reality of the upper sequence of subluminous stars apparently defined by the objects in Table I.

In the previous discussion (Eggen, 1969a) attention was called to two common proper motion systems in which one component may be an RSL object. The $(\mathrm{R}-\mathrm{I})$ photometry for these stars gives the following;

\begin{tabular}{llllllllll} 
Star & $\mathrm{V}_{E}$ & $\mathrm{~B}-\mathrm{V}$ & $\mathrm{U}-\mathrm{B}$ & $\mathrm{R}$ & $\mathrm{R}-\mathrm{I}$ & $\mathrm{N}, \mathrm{N} \mu$ & $\theta$ & \\
$\mathrm{G} \mathrm{22-9}$ & $10^{\mathrm{m}} 10$ & +0.705 & +0.15 & $9^{\mathrm{m}} .84$ & +0.26 & 2,3 & 0.305 & $192^{\circ}$ & LTT 7511 \\
\multicolumn{1}{c}{-8} & 13.52 & +0.96 & +0.33 & 12.97 & +0.41 & 4,3 & 0.305 & $192^{\circ}$ & LTT 7512 \\
& & & & & & & & & \\
LTT 5430 & 9.34 & +0.815 & +0.35 & 9.06 & +0.295 & 4,2 & 0.22 & 170 & \\
LTT 5428 & 13.98 & +0.70 & +0.20 & 13.58 & +0.23 & 3,3 & 0.23 & 268 &
\end{tabular}

The components of the first pair are separated by 33" and those of the second by 300 ". From the (R, I) photometry it appears that the first pair may consist of halo population subdwarfs with a parallax of 0.009 and tangential velocity of $160 \mathrm{~km} / \mathrm{sec}$. If this interpretation is correct, the radial velocity may be very large. If LTT 5430 is a normal, main sequence star the companion LTT 5428 , has $\mathrm{M}(\mathrm{I})$ near $+10^{\mathrm{m}} 2$ and is an RSL object with tangential velocity of $45 \mathrm{~km} / \mathrm{sec}$.

An additional common proper motion pair that deserves astrometric and spectroscopic attention is the following:

$\begin{array}{llllllll}\text { Star } & \mathrm{V}_{E} & \mathrm{~B}-\mathrm{V} & \mathrm{U}-\mathrm{B} & \mathrm{R} & \mathrm{R}-\mathrm{I} & \mu & \theta \\ \mathrm{G} 85-44 & 12^{\mathrm{m}} 48 & +1^{\mathrm{m}} 50 & +1.00 & 11^{\mathrm{m}} 28 & +1.04 & 0.61 & 171^{\circ} \\ \text { G85-40 } & 14.76 & +1.15 & +0.67 & 14.10 & +0.46 & 0.61 & 170^{\circ}\end{array}$

The components are separated by 1.2 . The fainter component is also G83-53 (0.63, $\left.172^{\circ}\right)$, and $\mathrm{G} 97-16\left(0.58,168^{\circ}\right)$ whereas the brighter component is also $\mathrm{G} 97-12$ $\left(0.61,167^{\circ}\right)$ and Ross $388\left(0.59,173^{\circ}\right)$. In spite of the large separation, the large common proper motion makes it almost certain that the stars are physically related. If the brighter star populates the Hyades main sequence the parallax is 0.039 , the tangential velocity is $75 \mathrm{~km} / \mathrm{sec}$ and the fainter component is a RSL star with $\mathrm{M}(\mathrm{I})=$ +11.6 at $(\mathrm{R}-\mathrm{I})=+0.46$, making it nearly identical to LTT 2236 in Table I. 


\section{References}

Altena, W. F. van: 1969, Astron. J. 74, 2.

Eggen, O. J.: 1969a, Astrophys. J. Suppl. 19, 31.

Eggen, O. J.: 1969b, Astrophys. J. 158, 1109.

Eggen, O. J.: 1970a, Astrophys. J. 159, 945.

Eggen, O. J.: 1970b, Astrophys. J., in press.

Eggen, O. J. and Greenstein, J. L. :1965, Astrophys. J. 141, 83.

Greenstein, J. L. and Eggen, O. J.: 1966, in Vistas in Astronomy (ed. by A. Beer), Pergamon Press, New York.

Joy, A. H.: 1947, Astrophys. J. 105, 96.

Luyten, W. J.: 1957, Catalogue of 9867 stars in the Southern Hemisphere with Proper Motion Exceeding $0^{\prime \prime} .2$ Annually, Lund Press, Minneapolis. 